\title{
A Novel Method for Defining Health Facility Catchment Areas in a Low Income Country
}

\author{
Kate Zinszer², Ruth Kigozi ${ }^{3}$, Katia Charland ${ }^{1}$, Dr. Grant Dorsey ${ }^{4}$, Dr. Moses Kamya ${ }^{5}$ and \\ David Buckeridge ${ }^{1}$
}

${ }^{1}$ McGill University, Montreal, QC, Canada; ${ }^{2}$ Harvard Medical School, Boston, MA, USA; ${ }^{3}$ Uganda Malaria Surveillance Project,

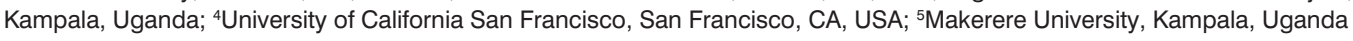

\section{Objective}

We propose a simple statistical method, the cumulative case ratio, for defining a catchment area using surveillance data.

\section{Introduction}

The catchment area of a health-care facility is used to assess health service utilization and calculate population-based rates of disease. Current approaches for catchment definition have significant limitations such as being based solely on distance from the facility or using an arbitrary threshold for inclusion.

\section{Methods}

The catchment areas of six health-care facilities in Uganda were determined using the cumulative case ratio: the ratio of the observed to expected utilization of a facility for a particular condition by patients from small administrative areas. The cumulative case ratio for malaria-related visits to these facilities was determined using data from the Uganda Malaria Surveillance Project. Catchment areas were also derived other methods such as the straight line and road network distances from the facility. Subsequently, the 1-year cumulative malaria case rate (the total number of cases during one year) was calculated for each catchment area, with at-risk populations estimate from catchment areas determined using the cumulative case ratio, the straight-line distance, and the road network distance.

\section{Results}

The 1-year cumulative malaria case rate varied considerably with the method used to define the catchment areas. With the cumulative case ratio approach, the catchment area could include noncontiguous areas. With the distance approaches, the denominator increased substantially with distance, whereas the numerator increased only slightly. The largest cumulative case rate per 1000 population was for the Kamwezi facility: 234.9 (95\% confidence interval, CI: $226.2-$ 243.8) for a straight-line distance of $5 \mathrm{~km}, 193.1$ (95\% CI: $186.8-$ 199.6) for the cumulative case ratio approach and 156.1 (95\% CI: 150.9-161.4) for a road network distance of $5 \mathrm{~km}$.

\section{Conclusions}

The variation in estimates of the cumulative rate of confirmed malaria cases was attributable to the differential change in the numerator and denominator of the case rate calculation as a function of the distance used to define the catchment area. An erroneous view of the catchment area can lead to inefficient and inadequate services, misspecification of the catchment population and potentially flawed decision-making on other facilities, such as deciding where to locate a new facility. Our approach is simple, reproducible, and is based on a statistical measure to decide which administrative units should be included in catchment areas.

\section{Keywords}

catchment; surveillance; Uganda
*Kate Zinszer

E-mail: kate.zinszer@mail.mcgill.ca 